\title{
Research on Immunity to Electric Impulsive Interference of ZPW-2000 Track Circuit in Station
}

\author{
Xiaochun Wu and Guoqing $\mathrm{Li}$ \\ School of Automation \& Electrical Engineering, Lanzhou Jiao Tong University, \\ Lanzhou 730070 P.R. China \\ 506270698@qq.com
}

\begin{abstract}
Taking the ZPW-2000 track circuit in station as the research object, to study the protection of electric impulsive interference. Firstly analyzing the making reasons and characteristics of the impulsive interference, and pointing out the shortage of chock transformer in impulsive interference protection. Then combining with the principle of anti-interference to improve the traditional chock transformer. Finally through the relative parameters comparison between traditional and improved chock transformer to prove the higher anti impulsive interference ability of the improved chock transformer, that can meet the requirements of anti-interference.
\end{abstract}

Keywords: Impulsive interference, Station ZPW-2000 track circuit, Chock transformer, Interference protection

\section{Introduction}

The station ZPW-2000 track circuit gets the track circuit information and the train vehicle information to gather, and sends this information to steel track at same time in any time[1]. Station track circuit uses mechanical insulated joint to segmente sections[2], and uses the choke transformer to ensure the successful proceeing of traction current. Considering the problem of unbalanced circuit on the steel track caused by traction current, station track circuit adopts the choke transformer with adaptation to reduce the interference of unbalanced traction current.

The choke transformer with adaptation was desiged for $25 \mathrm{~Hz}$ phase sensitive track circuit so far[3], and it cannot be used on station ZPW-2000 track circuit. So it is necessary to design a choke transformer for station ZPW-2000 track circuit to meet the anti-electric interference requirements of track circuit.

\section{Analysis Interference of Electric Impulsive Current}

The main transformer of locomotive may appear tremedous energizing inrush when locomotive passes the phase-separating section or rises, downs the bow and so on. This tremedous energizing inrush can reach 3 to 6 times larger than steady current[4], and can cause a great quantity of unbalanced traction impulsive current which contains the DC component and the higher harmonic component[5], so brings the following two aspects interference for station ZPW-2000 track circuit:

(1) The DC component and large AC component of impulsive current easily cause the magnetic saturation of choke transformer, resulting in the received voltage of frequency shift signal is too small, appearing frequency shift signal instantaneous collapse phenomenon, causing "flashing red" [6];

(2) The higher harmonic of implusive current that has the same frequency with carrier frequency may enter the singal receiver passband, causes the signal interference of inband, and effects the reliable transmission of singal. Taking carrier frequency 1700-1 as 
an example, the range of filter passing band is $1700-1 \pm 40 \mathrm{~Hz}$, due the unbalanced traction current with $50 \mathrm{~Hz}$ fundamental frequency can generate $1700 \mathrm{~Hz}$ higher harmonic interference current [7], The $1700 \mathrm{~Hz}$ higher harmonic interference current can enter the signal receiver passband and brings interference to in-band original frequency shift signal.

\section{Theoretical Analysis of Anti-Interference Method}

According to the characteristics of electric implusive interference and the antiinterference principle, the station ZPW-2000 track circuit impulsive interference protection scheme mainly takes measures from the following two aspects: (1) reducing the interference energy; (2) improving the equipment anti interference ability.

\subsection{Reduce Interference Energy}

The influence of electric impulsive interference for station ZPW-2000 track circuit is determined by the $50 \mathrm{~Hz}$ voltage that is generated by unbalanced traction current shock at both ends of the choke transformer, when the voltage exceeds $2.4 \mathrm{~V}$, the station track circuits will produce "red band", therefore, it is necessary to reduce the impulsive current voltage at both ends of choke transformer. Due to the size of impulsive current is determined mainly by the unbalance factors of rail and choke transformer, and has nothing with impedance of track circuit sender and receiver, so we can regard the interference source as a current source, the parallel circuit of receiver and choke transformer is treated as a load. According to the principle of anti-interference, when the interference source is a current source or an approximate current source, the series resonance circuit can be used in parallel circuit with the interference source to reduce the interference [8], the schematic diagram is shown in Figure 1.

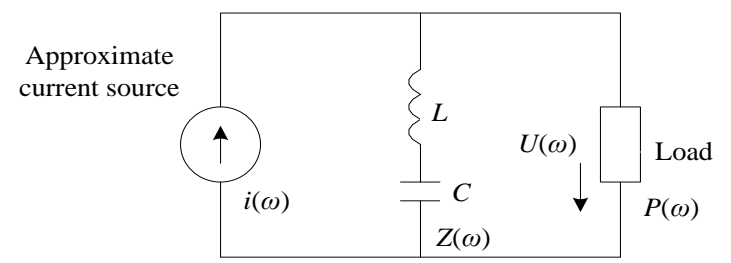

Figure 1. Flow Source Interference Reduction Principle Diagram

In Figure 1, $i(\omega)$ is the source of the interference current, when $\omega$ equals to the $\omega_{0}$ that is resonance circuit frequency, the $L C$ circuit forms series resonant, this resonant circuit impedance $z\left(\omega_{0}\right)$ is the smallest, the limit $z\left(\omega_{0}\right) \rightarrow 0$, so load disturbance voltage $u\left(\omega_{0}\right)=i\left(\omega_{0}\right) z\left(\omega_{0}\right) \rightarrow 0$, and interference power $p\left(\omega_{0}\right)=i\left(\omega_{0}\right) u\left(\omega_{0}\right) \rightarrow 0$. Obviously, the interference energy of the load is very small, and may approache zero in the ideal situation.

According to the above principle, the series resonant circuit of a $50 \mathrm{~Hz}$ can be paralleled with the impulsive interference source, and the schematic diagram is shown in Figure 2. $50 \mathrm{~Hz}$ series resonant circuit is paralleled in the secondary coil of the choke transformer. When there is a interference source, the choke transformer secondary series resonant circuit is equivalent to a wire, its resonant impedance $Z_{50}$ is very small and when converted to the impedance of traction circle its value is less, so the excitation current of flowing through the traction circle is very small, the interference voltage at both ends of the choke transformer is cut down, the limit is $0 \mathrm{~V}$, interference energy is reduced. 


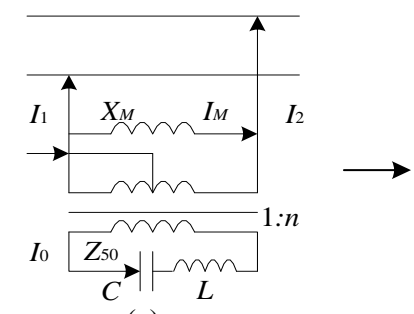

(a)

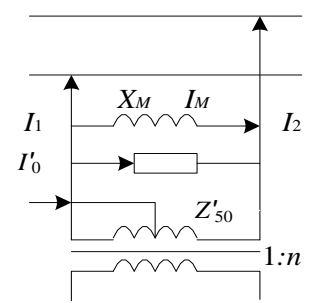

(b)

\section{Figure 2. Paralleling Series Resonant Circuit with Choke Transformer Secondary Coil}

In Figure 2(a), $Z_{50}$ represents $50 \mathrm{~Hz}$ series resonant impedance, $I_{0}$ is series resonant current, $X_{M}$ is magnetizing impedance of traction circle, $I_{M}$ is magnetizing current of traction circle; In Figure 2(b), $I_{0}^{\prime}$ is the current when $I_{0}$ in secondary coil is converted to the traction circle, $Z_{50}^{\prime}$ is the impedance when $50 \mathrm{~Hz}$ series resonant impedance in secondary coil is converted to the traction circle.

According to Figure 2 (a), the amplitude of exciting current $I_{M}$ can be obtained. $\left|\dot{I}_{M}\right|=\sqrt{\left(\dot{I}_{1}-\dot{I}_{2}\right)^{2}-\left(2 \dot{I}_{0} n\right)^{2}}$

Figure 2(b) is the equivalent circuit diagram of Figure 2(a), the amplitude of exciting current $I_{M}$ can be obtained in further.

$$
\left|\dot{I}_{M}\right|=\left|\frac{\left(\dot{I}_{1}-\dot{I}_{2}\right)}{2} \square \frac{\frac{Z_{50}}{n^{2}}}{\frac{Z_{50}}{n^{2}}+j X_{M}}\right|
$$

By the formula (2), when the series resonant impedance $Z_{50}$ decreases, the amplitude of exciting current in traction current also decreases, so the $50 \mathrm{~Hz}$ voltage at both ends of choke transformer decreases, which meets the require of reducing interference.

\subsection{Increase Anti Interference Ability of Choke transformer}

The anti-interference ability of the equipment plays a vital role in the interference protection scheme. Due the DC component and higher AC component of impulsive current are easy to cause the choke transformer magnetic saturation phenomenon, and affect the signal transmission. Therefore, it is necessary to prevent the choke transformer magnetic saturation. The usual method is to increase the iron core air gap of choke transformer [9], the principle of methods is as follows.

$L_{M}=\frac{N^{2} \mu S}{l}$

$L_{M}^{\prime}=\frac{N^{2} \mu_{0} S}{\delta}\left(1+\frac{\mu_{0} l}{\mu \delta}\right)^{-1}$

By increasing the iron core air gap to increase the choke transformer magnetic saturation current, formula (4) and (5) are inductance value of choke transformer with and without air gap respectively, $n$ is the number of turns of the coil, $S$ is the effective crosssectional area of the iron core, $\mu$ is iron permeability, $\mu_{0}$ is air permeability, $l$ is the average magnetic path length of iron core, $\delta$ is the length of the air gap. When $\mu_{0} l / \mu \delta<<1$, $L_{M}^{\prime}=n^{2} \mu_{0} \mathrm{~S} / \delta, L_{M}^{\prime} / L_{M}=\mu_{0} l / \mu \delta<<1$, the energizing inductance of traction circle decreases, and the $50 \mathrm{~Hz}$ impedance $X_{M}=j 2 \pi 50 L_{M}$ also decreases, so the choke transformer magnetic saturation current value increases. When the interference emerges, because the choke 
transformer magnetic saturation current increases, the choke transformer cannot occurred magnetic saturation phenomenon, signal can transfer credibly; the anti-interference ability of equipment is improved.

\subsection{Ensure Reliable Transmission of Frequency Shift Signal}

When through increasing iron core air gap to reduce the traction circle $50 \mathrm{~Hz}$ impedance, the frequency shift signal impedance is also reduced, which may lead the signal impedance of traction circle smaller than $17 \Omega$, and harming the ability of signal transmission. So it is necessary to guarantee the signal reliable transmission. Basing on the parallel resonant principle, the capacitance $C_{2}$ is designed to be paralleled with the secondary coil of the transformer. And $C_{2}, L C$ series resonance circuit and choke transformer coil all constitute parallel resonant for frequency shift signal, this parallel resonant plays high impedance for frequency shift signal. In this way the traction circle impedance converted from secondary side is more than $17 \Omega$ and the requirements of current signal transmission is met. The overall structure of anti-interference device is shown in Figure 3(a) and the connection of anti-interference device with the signal device is shown in Figure 3(b).

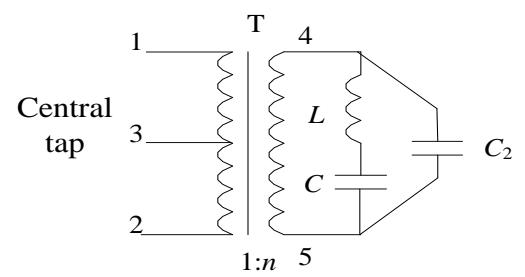

(a) Diagram of Anti Impulsive Interference Device

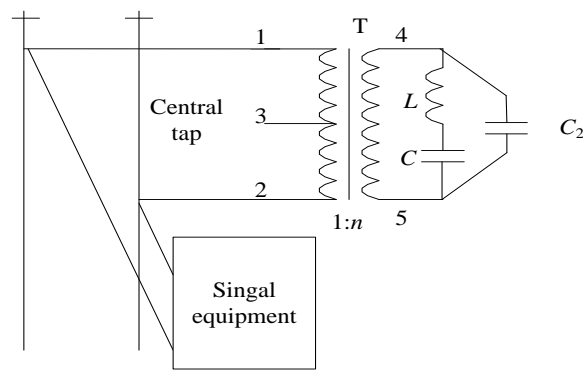

(b) Connection Diagram Between Anti Impulsive Interfrence Device and Signal Equipment

Figure 3. Diagram of Anti Impulsive Interference Device

\section{Parametric Solution}

On the basis of theoretical design structure, taking the station ZPW-2000 track circuit as the research object, to calculate the $50 \mathrm{~Hz}$ impedance, $L, C, C_{2}$ and the open air gap length $\delta$.

\subsection{The Solution of $50 \mathrm{~Hz}$ Impedance of Traction Circle}

Firstly analyzing the saturation characteristic of the traditional choke transformer. Only when $50 \mathrm{~Hz}$ steady-state unbalance current is imposed on the primary coil of the choke transformer, 2 groups of test data are obtained. Results are shown in Table 1. 
Table 1. Impedance Characteristic of Current Transformer

\begin{tabular}{cccccc}
\hline $\mathrm{U}_{1}(\mathrm{~V})$ & $\mathrm{I}_{1}(\mathrm{~A})$ & $\mathrm{Z}_{1}(\Omega)$ & $\mathrm{U}_{2}(\mathrm{~V})$ & $\mathrm{I}_{2}(\mathrm{~A})$ & $\mathrm{Z}_{2}(\Omega)$ \\
\hline 11.49 & 4.97 & 2.31 & 11.39 & 4.95 & 2.30 \\
20.59 & 10.0 & 2.06 & 20.2 & 10.15 & 1.99 \\
26.69 & 20.5 & 1.30 & 25.22 & 20.4 & 1.24 \\
29 & 30.0 & 0.97 & 26.86 & 29.9 & 0.90 \\
\hline
\end{tabular}

According to the data in Table 1, choke transformer appears magnetic saturation when the current in the primary coil is $10 \mathrm{~A}$, which equivalents to $20 \mathrm{~A}$ of the unbalance current, the corresponding impedance value is $2 \Omega$. If saturation is required not to emerge when the unbalance current is $100 \mathrm{~A}$, equivalents to $50 \mathrm{~A}$ current in primary coil, the choke transformer traction circle $50 \mathrm{~Hz}$ impedance after opening air gap should be reduced to $1 / 5$ of the original value, is $0.4 \Omega$, and the corresponding inductance value is $L_{M}=j 2 \pi 50 / X_{50 M}=1.27 \mathrm{mH}$.

\subsection{Solution of Parameters $C, L, C_{2}$}

The constraints for geting parameters $L, C, C_{2}$ are the following three aspects: (1) $L$ and $C$ constitute a $50 \mathrm{~Hz}$ series resonant circuit[10]; (2)The inductive reactance of $L C$ series resonant circuit, the excitation inductive reactance of choke transformer secondary coil and $C_{2}$ constitute the parallel resonance for frequency shift; (3) Consider the price and volume of the device.

By the simulation study of choke transformer can reach that choke transformer secondary coil equivalent inductance reactance is $L_{2}=2.88 \mathrm{H}$, which along with $L C$ series resonant circuit and $C_{2}$ constitute the parallel resonance for frequency shift,the principle of parallel resonant is shown in Figure (4).

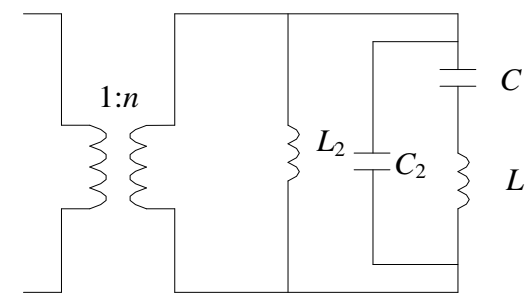

Figure 4. The Schematic Diagram of Parallel Resonance

According to the principle of parallel resonance can derive the following Formula (5):

$$
\frac{\frac{\omega_{2}^{2} L C-1}{\omega_{2} C} \times \omega_{2} L_{2}}{\frac{\omega_{2}^{2} L C-1}{\omega_{2} C}+\omega_{2} L_{2}}=\frac{1}{\omega_{2} C_{2}}
$$

In formula (5), $\omega_{2}=2 \pi f_{2}$ is the angular frequency of frequency shift signal carrier frequency, $f_{2}$ is the carrier frequency of frequency shift signal, the formula of calculating parameter is as follows.

$$
L=\frac{1}{\omega_{1}^{2} C}
$$




$$
C_{2}=\frac{1}{\omega_{2}^{2} L_{2}}+\frac{C}{\left(\frac{f_{2}}{f_{1}}\right)^{2}-1}
$$

Where $\omega_{1}=2 \pi f_{1}, C$ is a setting value, so must firstly determining the $C$, according to the price and the volume of capacitance, referencing the component parameter of the choke transformer with adaptation that applies to $25 \mathrm{~Hz}$ phase sensitive track circuit, taking $C=30 \mu \mathrm{F}$, so $L=0.338 \mathrm{H}$. $C_{2}$ is related to frequency of frequency shift signal, so using formula (7) to calculate the four different $C_{2}$ values are $28.9 \mathrm{nF}, 20.9 \mathrm{nF}, 15.8 \mathrm{nF}, 12.4 \mathrm{nF}$, which correspond the four different carrier frequency, which are $1700 \mathrm{~Hz}, 2000 \mathrm{~Hz}$, $2300 \mathrm{~Hz}, 2600 \mathrm{~Hz}$ in order.

\subsection{Solution of Length of Air Gap}

Increasing the magnetic saturation current by increasing the transformer iron core air gap, to avoid the choke transformers saturation phenomenon, this phenomenon is caused by larger interference current and affects signal transmission [11]. The value of iron core air gap that used in the choke transformer with adaptation which applies to $25 \mathrm{~Hz}$ phase sensitive track circuit is $0.5 \mathrm{~mm}$. And The electric impulsive interference environment of the ZPW-2000 station track circuit are similar to the electric impulsive interference environment of $25 \mathrm{~Hz}$ phase sensitive track circuit. Therefore, the value of iron core air gap referencing the adaptation of choke transformer parameters, get $\delta=0.5 \mathrm{~mm}$.

\section{Analysis of Anti-Impulsive Interference Ability of Improved Current Transformer}

\subsection{Hz Series Resonant Impedance}

The $50 \mathrm{~Hz}$ resonant impedance of the improved choke transformer should to be infinite small in the ideal situation[12], in fact, it is related to quality factor $Q$ of choke transformer, the value range of quality factor $Q$ is $20 \sim 30$,according to the formula (8)

$$
Q=\frac{1}{R} \cdot \sqrt{\frac{L}{C}}=\frac{\omega L}{R}=\frac{f_{0}}{\Delta f}
$$

The $50 \mathrm{~Hz}$ resonant impedance $R=\omega L / Q=2 \pi f L / Q$ is obtained. Setting $Q=20$ and $L=0.338 \mathrm{H}$, so $R=5.3 \Omega$. If the transformer turns ratio is set $n=30$, the equivalent impedance of the resonant circuit converted to the primary coil of the choke transformer is $R_{0}=R / n^{2}=0.0059 \Omega$, smaller than $0.01 \Omega$ that is technical indicator of the UM71 air core coil, meeting the requirements.

\subsection{Hz Excitation Current Comparison}

$50 \mathrm{~Hz}$ excitation current of traditional current transformer is $I_{M}$ :

$$
I_{M}=\left|\frac{\dot{I}_{1}-\dot{I}_{2}}{2}\right|
$$

$50 \mathrm{~Hz}$ excitation current of improved current transformer is $I_{0}^{\prime}$ :

$$
I_{0}^{\prime}=\left|\frac{\left(\dot{I}_{1}-\dot{I}_{2}\right)}{2} \times \frac{Z_{50}^{\prime}}{Z_{50}^{\prime}+j X_{M 50}}\right|=0.0145\left|\frac{\dot{I}_{1}-\dot{I}_{2}}{2}\right|
$$

In formula (10), $Z_{50}=0.0044 \Omega, X_{M 50}=0.4 \Omega$. Comparing formula (9) and formula (10): $\left|I_{0}^{\prime} / I_{M}\right|=68.9$. It is showed that excitation current is reduced greatly. 


\subsection{The Contrasting of Interference Voltages and Interference Power of Traction Circle}

The traditional choke transformer traction circle electric interference voltage and power:

$$
\begin{gathered}
U_{n}^{\prime}=\left|\frac{\left(\dot{I}_{1}-\dot{I}_{2}\right)}{2} \times X_{M}^{\prime}\right|=1.6\left|\frac{\dot{I}_{1}-\dot{I}_{2}}{2}\right| \\
P_{n}^{\prime}=\left|\frac{\left(\dot{I}_{1}-\dot{I}_{2}\right)^{2}}{2} \times X_{M}^{\prime}\right|=1.6\left|\frac{\left(\dot{I}_{1}-\dot{I}_{2}\right)^{2}}{2}\right|
\end{gathered}
$$

The improved choke transformer traction circle electric interference voltage and power:

$$
\begin{gathered}
U_{n}=\left|\frac{\left(\dot{I}_{1}-\dot{I}_{2}\right)}{2} \times \frac{Z_{50}^{\prime} \cdot j X_{M 50}}{Z_{50}^{\prime}+j X_{M 50}}\right|=0.0044\left|\frac{\mid \dot{I}_{1}-\dot{I}_{2}}{2}\right| \\
P_{n}=\left|\frac{\left(\dot{I}_{1}-\dot{I}_{2}\right)^{2}}{2} \times \frac{Z_{50}^{\prime} \cdot j X_{M 50}}{Z_{50}^{\prime}+j X_{M 50}}\right|=0.0044\left|\frac{\left(\dot{I}_{1}-\dot{I}_{2}\right)^{2}}{2}\right|
\end{gathered}
$$

In the formula, the value of $X_{M 50}^{\prime}$ is $1.6 \Omega$ that is the equipment standard of current transformer. By the contrasting value $\left|U_{n}^{\prime} / U_{n}\right|=\left|P_{n}^{\prime} / P_{n}\right|=364$, the interference voltages and power is significantly reduced and the interference is reduced correspondingly.

\section{Conclusion}

Concerning the problem of station ZPW-2000 track circuit impulsive interference, firstly analyzing the impulsive interference comprehensively, then using the series resonant of impulsive interference, the parallel resonant of frequency shift signal and the equivalent circuit of choke transformed to improve traditional choke transformer. Finally, through the theoretical analyzing for improved choke transformed to show that interference current, interference voltage and interference power is greatly reduced, the transmission of the signal is more reliable, so that the train becomes safer.

\section{References}

[1] J. X. Ding and X. H. Miao, "Design of Chain Test Table for Station ZPW-2000A Type Frequency Shift Track Circuit", Railway Singal \& Communication, vol. 49, no. 11, (2013), pp. 43-44.

[2] M. X. Shi, "Discussion of Engineering Application for Station Integrated Track Circuit", Technology Innovation, vol.6, (2008), pp.9-13.

[3] X. Zhao, "Research on Unbalanced Traction Current of Traction Power Supply System", Beijing Jiaotong University, (2011).

[4] S. J. Wang, "Protection of Track Circuit to Interference of Electric Traction", Harbin Railway Science \& Technology, vol. 4, (2000), pp. 19-20.

[5] X. P. Li, "Research on Iinterference of Unbalanced Traction Current on Track Circuit", Beijing Jiaotong University, (2011).

[6] J. Y. Chen, X. L. Chen, "Technology and Application of 25Hz Phase Sensitive Track Circuit", China railway publishing house, BeiJing(2012).

[7] Z. W. Huang, X. Y. Wei and Z. Liu, "Extracting the Characteristic Frequency of High Asymmetric Pluse Disturbed by Impluse", Journal of the China Railway Society, vol. 34, no. 12, (2012), pp. 71-75.

[8] Y. Q. Kong and Y. Y. Wang, "Research on Anti-interference Methods about Unbalanced Traction Current on Track Circui", Electronic quality, vol. 1, (2014), pp. 22-24.

[9] K. Q. Wei, “Analysis of Choke Transformer Magnetic Saturation and Preventive Measures", 
Railway Singal \& Communication, vol. 3, (1995), pp. 26.

[10] G. Y. Qiu and X. J. Luo, “Circuit”, Higher Education Press, BeiJing(2008)

[11] M. G. Cao, "Research of Modeling and Test on Impedance Bond in the High Speed Railway", Beijing Jiaotong University, (2014).

[12] Y. Q. Wang, "Study on Interference Analysis and Protection of Traction Return Current to Signal Control System in Electrified Railway”, LanZhou Jiaotong University, (2012).

\section{Authors}

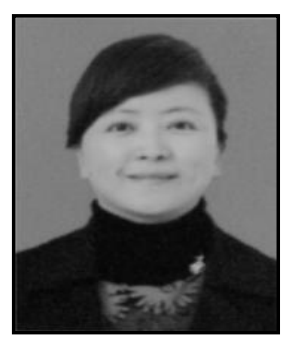

Xiaochun Wu, She received her master's degree in School of Automation \& Electrical Engineering from Lanzhou Jiaotong University, She is a associate professor at Lanzhou Jiaotong University. Her research interests include digital signal processing, wayside signaling Train Running Control System.

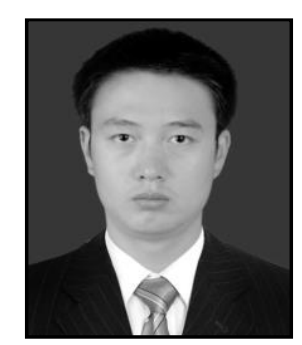

Guoqing Li, He is a master's student in School of Automation \& Electrical Engineering from Lanzhou Jiaotong University. His reacher interests include electric impulsive interference of ZPW2000 track circuit in station and digital signal processing. 